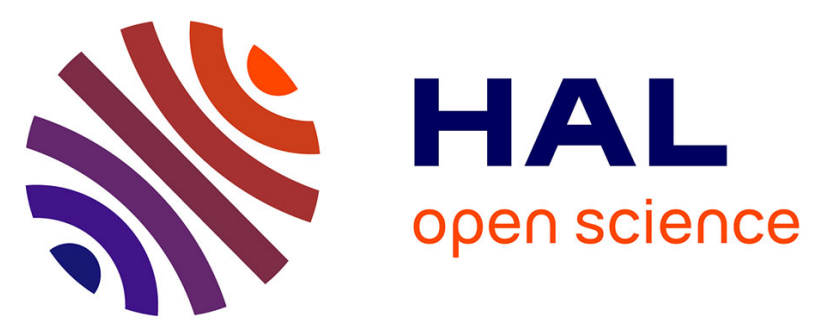

\title{
Analyzing the Influence of the Angles of Incidence and Rotation on MBU Events Induced by Low LET Heavy Ions in a 28-nm SRAM-Based FPGA
}

\author{
Jorge Tonfat, Fernanda Lima Kastensmidt, Laurent Artola, Guillaume
} Hubert, Nilberto H. Medina, Nemitala Added, Vitor A.P. Aguiar, Fernando Aguirre, Eduardo L.A. Macchione, Marcilei A.G. Silveira

\section{To cite this version:}

Jorge Tonfat, Fernanda Lima Kastensmidt, Laurent Artola, Guillaume Hubert, Nilberto H. Medina, et al.. Analyzing the Influence of the Angles of Incidence and Rotation on MBU Events Induced by Low LET Heavy Ions in a 28-nm SRAM-Based FPGA. IEEE Transactions on Nuclear Science, 2017, 64 (8), pp.2161-2168. 10.1109/TNS.2017.2727479 . hal-01705202

\section{HAL Id: hal-01705202 \\ https://hal.science/hal-01705202}

Submitted on 19 Feb 2018

HAL is a multi-disciplinary open access archive for the deposit and dissemination of scientific research documents, whether they are published or not. The documents may come from teaching and research institutions in France or abroad, or from public or private research centers.
L'archive ouverte pluridisciplinaire HAL, est destinée au dépôt et à la diffusion de documents scientifiques de niveau recherche, publiés ou non, émanant des établissements d'enseignement et de recherche français ou étrangers, des laboratoires publics ou privés. 


\title{
Analyzing the Influence of the Angles of Incidence and Rotation on MBU Events Induced by Low LET Heavy Ions in a 28-nm SRAM- based FPGA
}

\author{
Jorge Tonfat, Fernanda Lima Kastensmidt, Laurent Artola, Guillaume Hubert, Nilberto H. Medina, \\ Nemitala Added, Vitor A. P. Aguiar, Fernando Aguirre, Eduardo L. A. Macchione, Marcilei A. G. \\ Silveira
}

\begin{abstract}
This work shows the impact of low LET (Linear Energy Transfer) heavy ions on the reliability of 28-nm Bulk SRAM (Static Random Access Memory) cells from Artix-7 FPGA (Field-Programmable Gate Array). Irradiation tests on the ground showed significant differences in the MBU (Multiple Bit Upset) cross section of configuration (CRAM) and Block (BRAM) memory cells under various angles of incidence and rotation of the device. Experimental data are analyzed at transistor level by using the SEE (Single Event Effect) prediction tool called MUSCA SEP3 (MUlti-SCAle Single Event Phenomenon Prediction Platform) coupled with SPICE simulations.
\end{abstract}

Index Terms - FPGA, MBU, Soft Errors, Single Event Effects, layout analysis.

\section{INTRODUCTION}

$\mathrm{R}$ ELIABILITY of microelectronic CMOS (Complementary Metal Oxide Semiconductor) devices, such as soft errors, is a major concern especially for very integrated technologies such as SRAM (Static Random Access Memory)-based FPGAs (Field Programmable Gate Arrays). Then, it is critical to characterize their sensitivity to Single Event Upset (SEU) and Multiple Bit Upset (MBU). An MBU is defined as multiple bit upsets within a logical word of the memory. In this paper, the impact of low LET particles such

This work was supported in part by CNPq, CAPES, FAPERGS and FAPESP Brazilian Research Agencies.

J. Tonfat is with the Space Research Institute of the Austrian Academy of Sciences, Graz, A-8042, Austria and Instituto de Informática, PGMICRO, UFRGS, Porto Alegre 91501-970, Brazil (e-mail: jorge.tonfat@ oeaw.ac.at).

F. L. Kastensmidt is with Instituto de Informática, PGMICRO, UFRGS, Porto Alegre 91501-970, Brazil (e-mail: fglima@ inf.ufrgs.br).

L. Artola and G. Hubert are with The French Aerospace Lab (ONERA), Toulouse, France (email: firstname.name@onera.fr).

N. H. Medina, N. Added, V. A. P. Aguiar, E. L. A. Macchione and F. Aguirre are with Universidade de São Paulo, São Paulo, Brazil. e-mail: \{medina, nemitala, vpaguiar, macchion, faguirre\}@if.usp.br.

M. A. G. Silveira is with Centro Universitário da FEI, São Bernardo do Campo, Brazil. e-mail: marcilei@fei.edu.br. as heavy ions in a 28-nm Bulk SRAM FPGA from Xilinx (Artix-7) is highlighted. MBU in CRAM and BRAM are analyzed.

Recent work [1], revealed the impact of the angle of incidence of low LET ions on the SEU and MBU sensitivity of 28-nm SRAM-based FPGA. In this work, the main contribution is to propose new data and comparisons simulations with the aim to analyze in details the competition of the influence of the angle of incidence and rotation of low LET heavy ion particles on the occurrence of single and multiple bit upset in the same 28-nm SRAM-based FPGA. Results show that the knowledge of the bitstream logical organization is needed to determine with relevance SEUs and MBUs in the FPGA memories. MBU data is more relevant for the FPGA user since the physical organization of the memory cells is usually unknown in these devices. These new results give us the required information to apply the most effective detection and correction mechanism to deal with multiple upsets in CRAM and BRAM. The analysis of MBU events in SRAM-based FPGAs is more complex than in standard SRAM memories since the current architectures are composed of heterogeneous blocks composed with different schemes and layouts [2].

Recent work [1] compared experimental data with simulations performed at transistor level by the prediction tool MUSCA SEP3 [3-5]. As published by Xilinx the SRAMbased FPGA (Artix-7) has been designed with HPL. Because of confidential reasons, specific processes, such as HPL, can be not reachable with the aim to investigate by simulation the SEE sensitivity of such SRAM based.

In the last session of this work, the results showed a very good agreement between the prediction and the measurements, as well the comparisons with previous publications. The LP process calibrated in this work can be used with the aim to investigate the SEE sensitivity of Xilinx FPGA Artix-7; for this reason, the LP process have been used to analyze the effect of DUT orientation presented in experimental results. In this work, the effect of DUT orientation presented in experimental results is confirmed and analyzed with the simulation tool. 


\section{EXPERIMENT SETUP AND METHOdOLOGY TO ClASSIFY SEUS AND MBUS}

\section{A. Setup measurement of SEU/MBU under heavy ions}

The device under test (DUT) is a 28-nm Artix-7 FPGA from Xilinx. The device package was thinned to allow that heavy ions reach the active region. This FPGA is mounted into a low-cost commercial development board, Nexys 4 from Digilent [6]. The device was tested at nominal voltage $(1.0 \mathrm{~V})$. The Artix-7 FPGA is an array of configurable resources such as CLBs (Lookup Tables (LUTs) and Flip-Flops), DSPs, embedded block RAMs (BRAMs), and other specialized blocks.

These resources are configured and interconnected using a configuration memory based on SRAM cells. CRAM represents the largest percentage of the total SRAM cells in the device. The second largest memory is the one dedicated for the user circuit (BRAM). These two memories are logically organized in small segments named frames. In this device, the frames are composed of SRAM cells that are physically interleaved to minimize the occurrence of multiple bit upsets within the frame. In the case of the CRAM, the frames are protected with SECDED (single error correction and double error detection). As presented in [1], in the specific part tested (XC7A100T-CSG324), each frame has 3,232 bits and the CRAM has nearly 23 million bits and almost 6 million bits of BRAM. The analysis of both memories is presented independently because each memory is composed of different types of SRAM cells [7].

The heavy ions tests were performed at the Laboratório Aberto de Física Nuclear of the Universidade de São Paulo (LAFN-USP), Brazil in December 2015 and April 2016 [8]. The ion beams were produced and accelerated by the São Paulo 8UD Pelletron Accelerator. Table I summarizes the species, the angle of incidence $(\varphi)$ and the rotation angle $(\theta)$ of the board, the effective LET and the fluence. These tests were performed in a vacuum chamber and the effective LET for the tested angles are below $10 \mathrm{MeV} \cdot \mathrm{cm}^{2} \cdot \mathrm{mg}^{-1}$. The SRIM software was used to calculate the effective LET at the active layer [9]. The rotation of the board and the beam incident angle are depicted in Fig. 1a. The board containing the DUT is connected to a control computer through a USB interface for JTAG communication. An external power supply completes the test setup as shown in Fig. 1b.

In the control computer, custom-made software is responsible for the data collection. The test performs a semistatic approach and consists on loading a known bitstream before the beam is on and then continuously readback the stored bitstream to capture the upsets. The configuration and readback of the FPGA is done through the Xilinx iMPACT tool [10]. Then the readback bitstream is compared against the original bitstream and to the last readback bitstream. In this way, the total accumulated and the last occurred upsets are logged.

\section{B. Method to classify SEUs and MBUs}

As presented in [1], once we have the readback bitstream

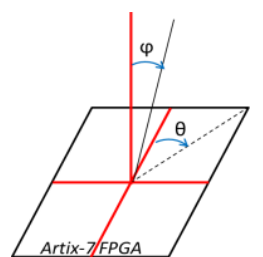

(a)

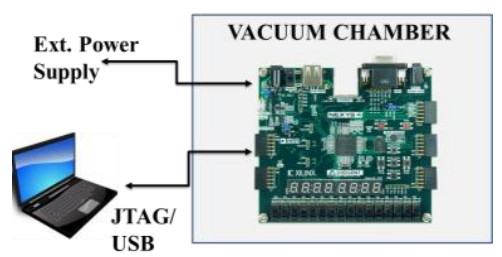

(b)
Fig. 1. In (a) Beam incident angle $(\varphi)$ and rotation angle $(\theta)$ of the board in the vacuum chamber. In (b) the test setup for heavy ion testing.

TABLE I

HEAVY ION RADIATION TEST PARAMETERS

\begin{tabular}{|c|c|c|c|c|c|c|}
\hline Run & Ion & $\begin{array}{l}\varphi \\
\left.0^{\circ}\right)\end{array}$ & $\begin{array}{r}\theta \\
\left(^{\circ}\right)\end{array}$ & $\begin{array}{r}\text { Energy } \\
(\mathrm{MeV})\end{array}$ & $\begin{array}{r}\text { Eff. LET } \\
\left(\mathrm{MeV} \cdot \mathrm{cm}^{2} \cdot \mathrm{mg}^{-1}\right)\end{array}$ & $\begin{array}{r}\text { Fluence } \\
(\text { particles } \\
\left./ \mathbf{c m}^{2}\right) \\
\end{array}$ \\
\hline 1 & ${ }^{12} \mathrm{C}$ & 0 & 0 & 50 & 3.00 & $2.21 \times 10^{6}$ \\
\hline 2 & ${ }^{12} \mathrm{C}$ & 45 & 0 & 50 & 5.21 & $4.96 \times 10^{5}$ \\
\hline 3 & ${ }^{12} \mathrm{C}$ & 60 & 0 & 50 & 9.13 & $8.50 \times 10^{5}$ \\
\hline 4 & ${ }^{7} \mathrm{Li}$ & 60 & 90 & 20 & 3.26 & $2.14 \times 10^{6}$ \\
\hline 5 & ${ }^{11} \mathrm{~B}$ & 45 & 90 & 42 & 3.74 & $1.76 \times 10^{6}$ \\
\hline 6 & ${ }^{12} \mathrm{C}$ & 0 & 90 & 28 & 5.25 & $1.52 \times 10^{6}$ \\
\hline 7 & ${ }^{11} \mathrm{~B}$ & 60 & 90 & 42 & 6.74 & $1.05 \times 10^{6}$ \\
\hline 8 & ${ }^{19} \mathrm{~F}$ & 0 & 90 & 52 & 8.26 & $5.19 \times 10^{5}$ \\
\hline 9 & ${ }^{16} \mathrm{O}$ & 45 & 90 & 60 & 9.57 & $5.59 \times 10^{5}$ \\
\hline
\end{tabular}

from the radiation tests, we can assign each upset to its correspondent configuration frame. So, when more than one upset appears in the same configuration frame, this event is classified as an MBU. The other cases are classified as SEUs. According to the byte position in the bitstream it is also possible to classify the upset as a CRAM frame or BRAM frame upset.

The adopted method to detect SEUs and MBUs implies that the contained data from the readback has truly MBUs generated by a single particle and not "constructed MBUs" generated from Coincident SEUs (CSEUs) from different particles. So the accuracy must be evaluated. In Table II is presented the details of the readback data obtained from the heavy ion test.

The ion flux was adjusted in each run to reduce the number of upsets per readback and in consequence the number of CSEUs is also reduced. It was used the same method proposed in [11] and [12] to evaluate the probability of CSEUs as a function of the percentage of the FPGA memory with upsets. The procedure combines Monte Carlo simulations and an analytical approach to estimate the probability. The Monte Carlo simulations consisted on defining a matrix with the same size of the Artix-7 tested part and then simulates the occurrences of random single upsets in the matrix up to the point where two of them construct an MBU. This simulation was run for 1,600,000 times for statistical significance. These data was plotted in Fig. 2 as a cumulative distribution function.

The other plotted curve corresponds to the analytical approach presented in [12]. Using a modified version of the birthday problem, this work calculates the probability of CSEUs in a readback data in function of the size of the matrix and the number of upsets in the readback as shown in eq. 1 . 
TABLE II

READBACK DATA CHARACTERISTICS

\begin{tabular}{crcr}
\hline \hline & & CRAM bits & \multicolumn{1}{c}{ BRAM bits } \\
\cline { 3 - 4 } Run & $\begin{array}{c}\# \\
\text { Readbacks }\end{array}$ & $\begin{array}{c}\text { Average upsets per } \\
\text { readback (\% of total } \\
\text { bits) }\end{array}$ & $\begin{array}{c}\text { Average upsets per } \\
\text { readback (\% of total } \\
\text { bits) }\end{array}$ \\
\hline 1 & 1019 & $34.53\left(1.49 \times 10^{-4}\right)$ & $10.85\left(1.88 \times 10^{-4}\right)$ \\
2 & 483 & $19.88\left(8.56 \times 10^{-5}\right)$ & $5.82\left(1.01 \times 10^{-4}\right)$ \\
3 & 609 & $28.71\left(1.24 \times 10^{-4}\right)$ & $7.88\left(1.37 \times 10^{-4}\right)$ \\
4 & 313 & $37.95\left(1.63 \times 10^{-4}\right)$ & $3.18\left(5.52 \times 10^{-5}\right)$ \\
5 & 695 & $25.16\left(1.08 \times 10^{-4}\right)$ & $4.86\left(8.43 \times 10^{-5}\right)$ \\
6 & 958 & $41.08\left(1.77 \times 10^{-4}\right)$ & $9.42\left(1.64 \times 10^{-4}\right)$ \\
7 & 534 & $25.33\left(1.09 \times 10^{-4}\right)$ & $4.52\left(7.85 \times 10^{-5}\right)$ \\
8 & 491 & $36.43\left(1.57 \times 10^{-4}\right)$ & $8.25\left(1.43 \times 10^{-4}\right)$ \\
9 & 792 & $16.96\left(7.31 \times 10^{-5}\right)$ & $3.65\left(6.34 \times 10^{-5}\right)$ \\
\hline \hline
\end{tabular}

$$
\operatorname{Prob}_{K}(n, p)(\text { collision }) \approx 1-\mathrm{e}^{\frac{-\mathrm{p}(\mathrm{p}-1)(2 \mathrm{k}-1)}{2 n}}
$$

Where $n$ is the size of the FPGA memory, $p$ is the number of upsets per readback and $k$ is named "collision range". The collision range was set to 1616 (half of the size of a frame), $p$ was defined as a vector where the minimum and maximum value where defined by the correspondent minimum and maximum upsets found per readback in our heavy ion tests. Both plots agree almost perfectly and they determine that for our average number of upsets per readback shown in Table II, the readback data have the probability of less than $8 \%$ of CSEUs.

\section{RESUltS OF SEU/MBU MEASUREMENTS}

In this section is presented two different analyses with different objectives. In the first analysis, the goal is to explore the increase of effective LET by changing the incident angle. In the second analysis, the goal is to explore the effect of the incident angle in similar effective LETs.

For each analysis the results are presented in terms of cross section. The error bars represent a 95\% confidence interval of upset counts using Poisson statistics. For the data points where statistical error bars are not shown, they are smaller than the size of the plotting symbols. When the organization of the memories (frames) is not taken into account, the cross section is presented as total upset cross section. On the other hand, the organization of the memories allows classifying the cross section into SEU, MBU or SEU+MBU cross section.

\section{A. Effect of incident angle at fixed particle energy}

This analysis corresponds to runs 1,2 and 3. The energy of the particle was fixed and three incident angles $(\varphi)$ were tested: $0^{\circ}, 45^{\circ}$ and $60^{\circ}$ which gave three different effective LETs: 3, 5.21 and $9.13 \mathrm{MeV} . \mathrm{cm}^{2} . \mathrm{mg}^{-1}$. The rotation angle is fixed to $0^{\circ}$. The cross section plots are presented in Fig. 3. It is presented the total upset, SEU, MBU and SEU + MBU cross sections for CRAM and BRAM memories.

It is observed that the cross section increases with the increase of effective LET as expected. Moreover, the MBU

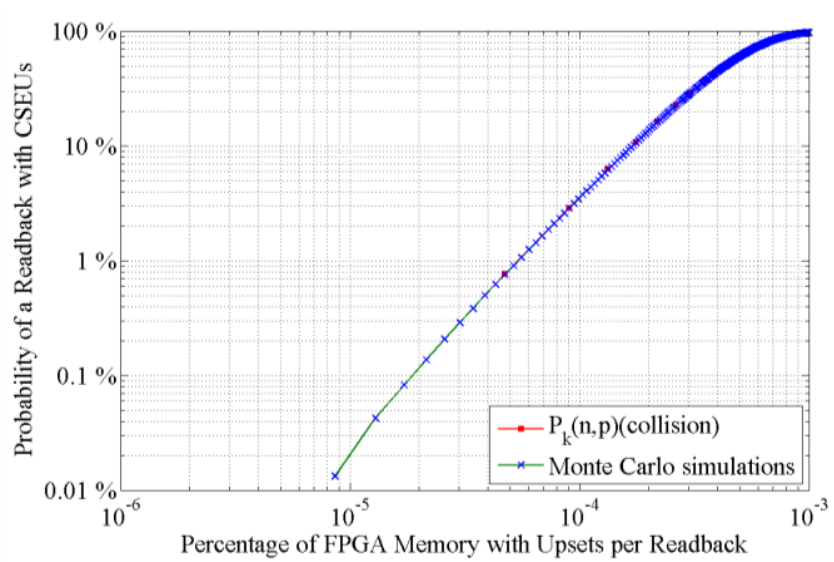

Fig. 2. Probability of CSEUs as function of percentage of FPGA memory with upsets.

cross section presents a higher increase than the other three classifications. The SEU cross section is higher than the MBU cross section for all the incident angles. The SEU + MBU cross section is similar to the total upset cross section because the upsets are dominated by the SEUs events.

\section{B. Effect of incident angle at similar LET}

This analysis evaluates runs 4 to 9 . From Table I, it is possible to observe, three different scenarios that are around to LET $=3.3$, LET $=5.6$ and LET $=9 \mathrm{MeV} \cdot \mathrm{cm}^{2} \cdot \mathrm{mg}^{-1}$. We tested 3 different incident angles: $0^{\circ}, 45^{\circ}$, and $60^{\circ}$. However, the rotation angle is fixed to $90^{\circ}$ and for each incident angle we tested only 2 different LETs. The cross section plots are presented in Fig. 4. It is presented the total upset, SEUs and MBUs cross sections. Similar to the first analysis, the SEU cross section is higher than MBU cross section. When Effective LET increases, the cross section increases (SEU and MBU) in all cases except MBU cross section of BRAM. When incident angle increases, the SEU and MBU cross section decrease for similar LETs.

When comparing the CRAM and BRAM MBU cross section, the latter is higher. Some hypotheses to the different susceptibility are that these SRAM cells are designed with different objectives. The CRAM cells are sensed directly and the content in principle should be static until the next reconfiguration. On the contrary, BRAM cells share the same bit lines and need to be optimized for writing and reading.

Finally, comparing the BRAM MBU cross section at rotation angles of $0^{\circ}$ and $90^{\circ}$ (Fig. $3 \mathrm{f}$ and Fig. 4f), the BRAM MBU cross section is higher at $\theta=90^{\circ}$. One possible explanation is that presumably the arrangement of BRAM cells is not the same in the row and column direction. A previous generation of SRAM-based FPGA (Virtex-5) presented similar effects to angles of incidence and rotation irradiation [15]. 


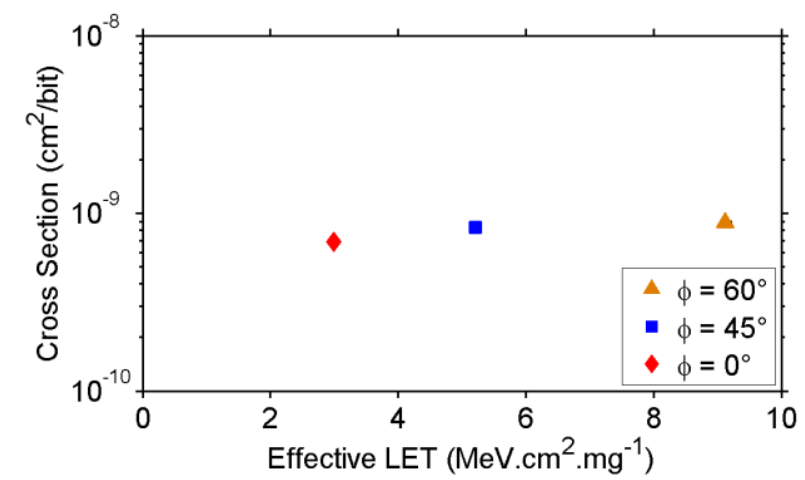

(a) Total Upsets Cross Section (CRAM)

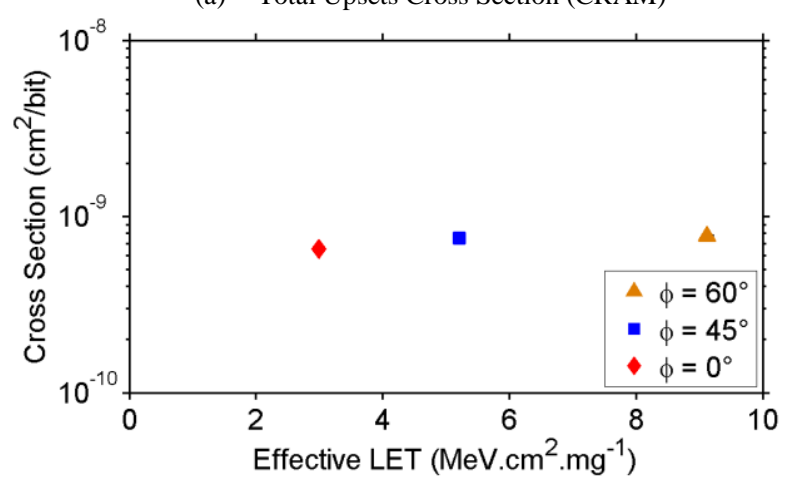

(c) SEU Cross Section (CRAM)

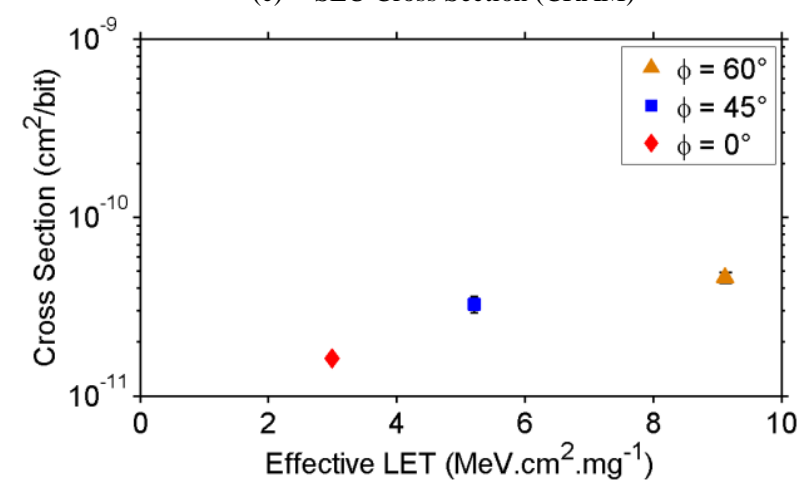

(e) MBU Cross Section (CRAM)

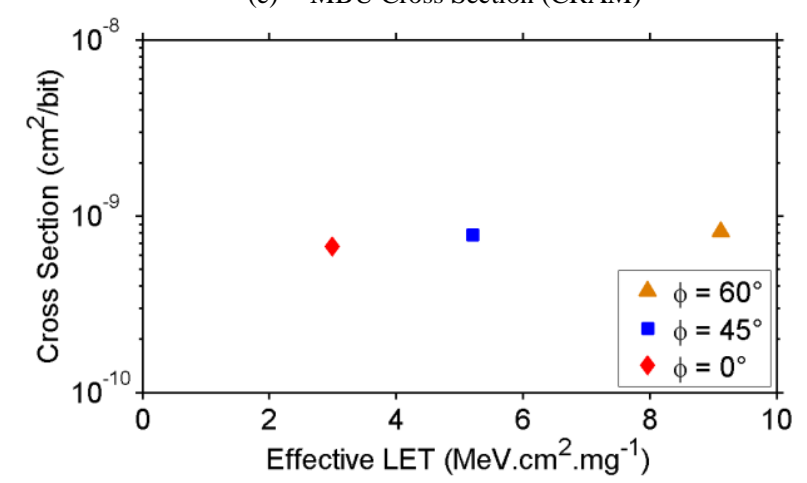

(g) $\mathrm{SEU}+\mathrm{MBU}$ Cross Section (CRAM)

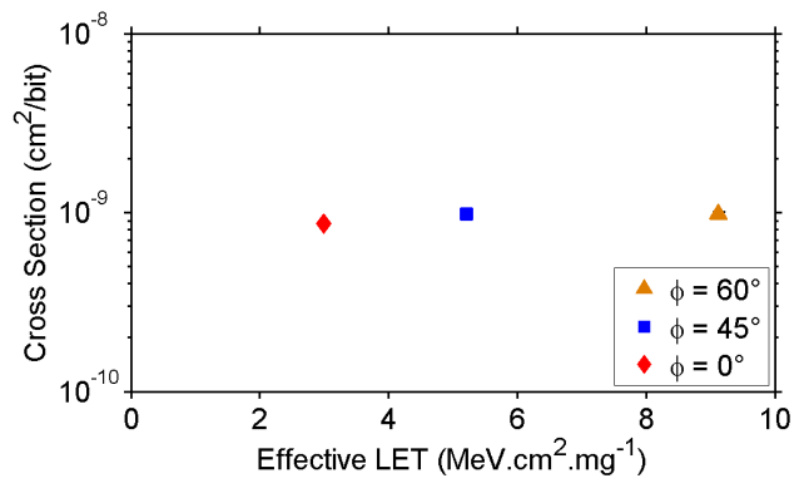

(b) Total Upsets Cross Section (BRAM)

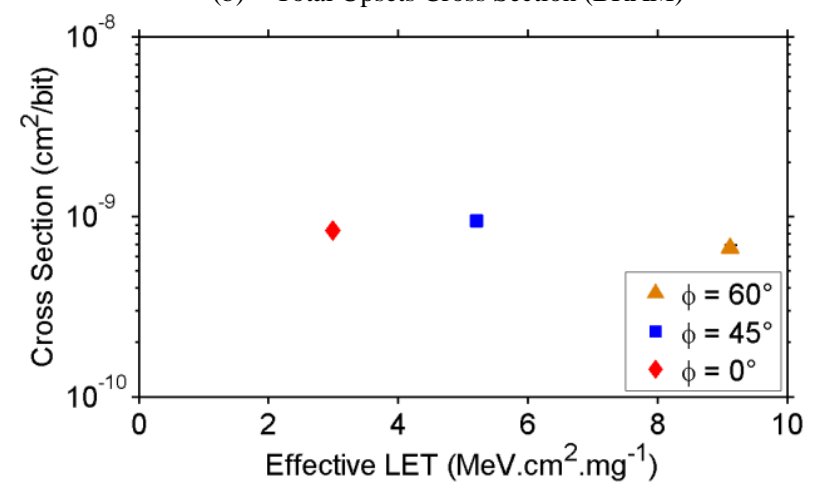

(d) SEU Cross Section (BRAM)

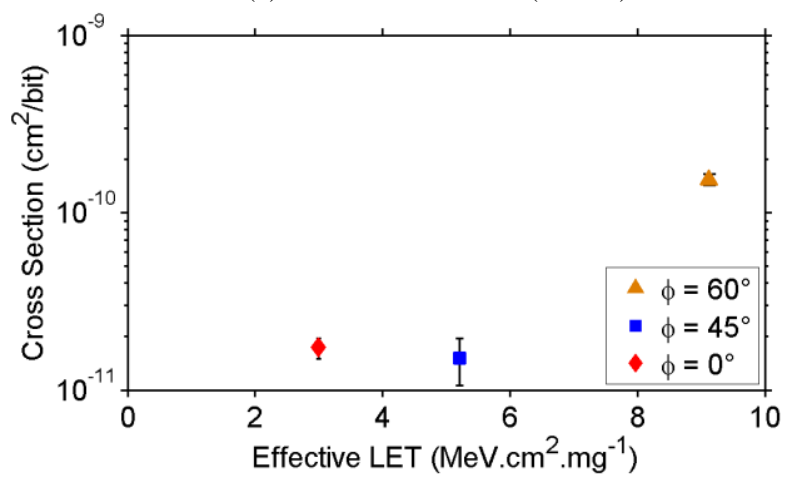

(f) MBU Cross Section (BRAM)

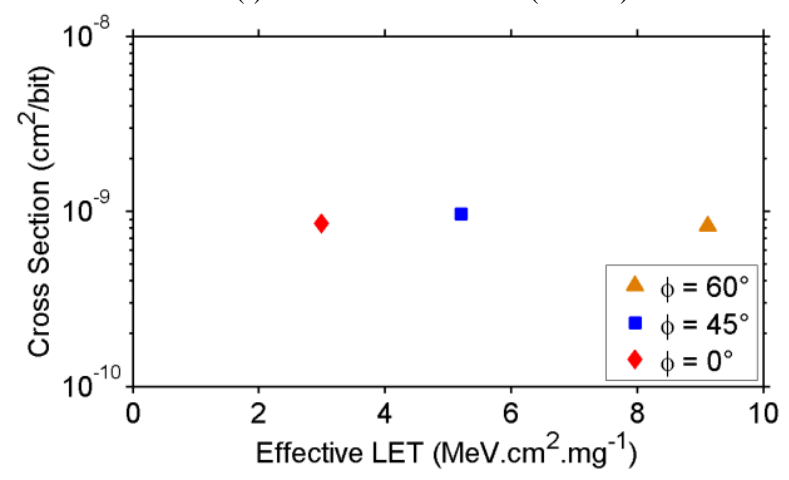

(h) SEU + MBU Cross Section (BRAM)

Fig. 3. Results of the first analysis campaign where the goal is to explore the increase of effective LET by changing the incident angle [1]. 


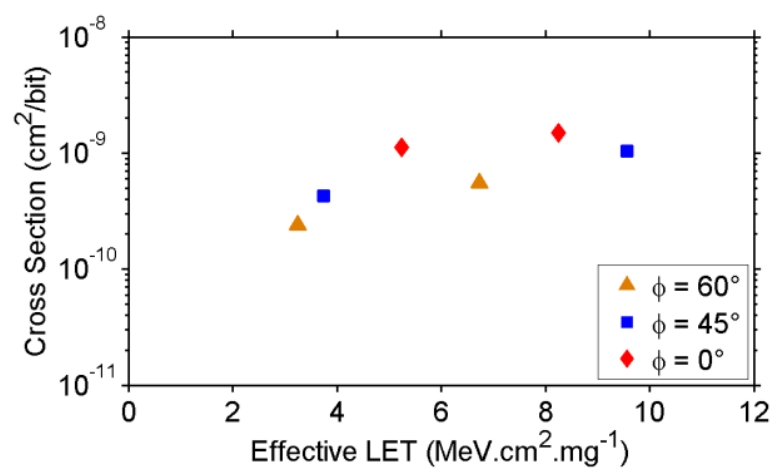

(a) Total Upsets Cross Section (CRAM)

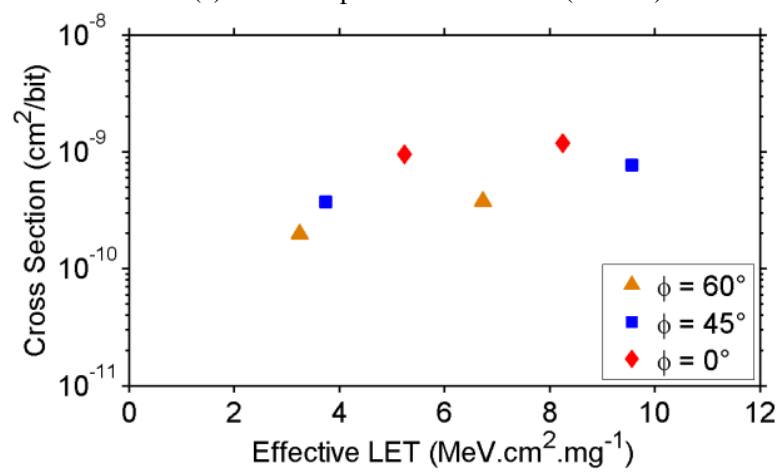

(c) SEU Cross Section (CRAM)

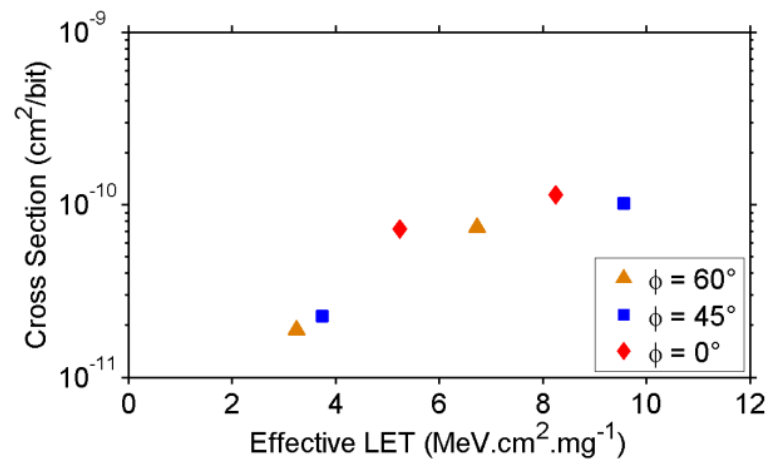

(e) MBU Cross Section (CRAM)

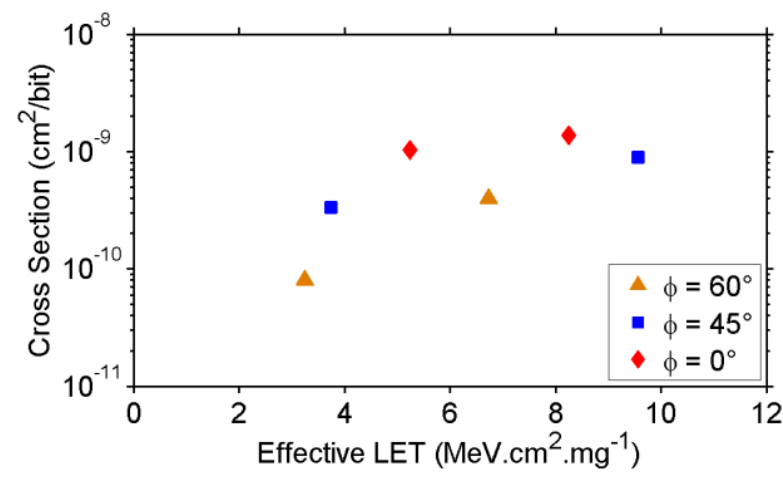

(b) Total Upsets Cross Section (BRAM)

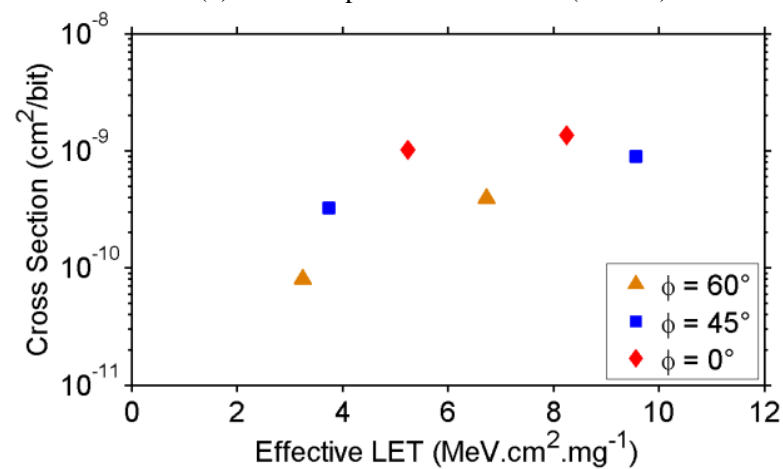

(d) SEU Cross Section (BRAM)

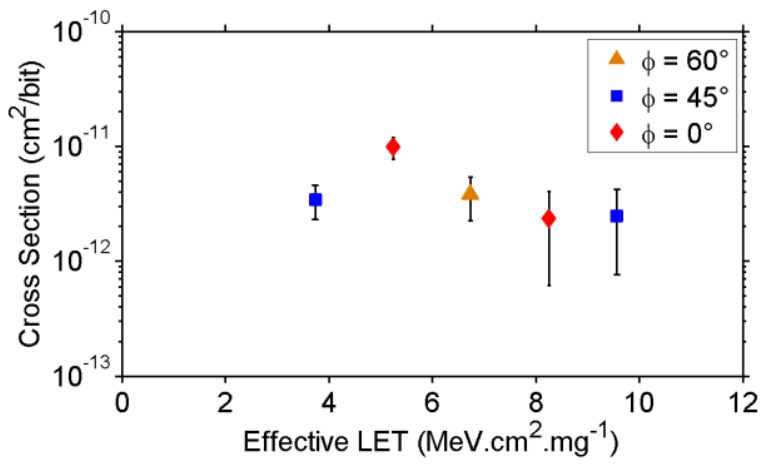

(f) MBU Cross Section (BRAM)

Fig. 4. Results of the second analysis campaign where the goal is to explore the effect of the incident angle in similar effective LET. Plot (f) presents only 5 cross sections because run $4\left({ }^{7} \mathrm{Li}, \phi=60^{\circ}\right)$ presented no MBU events for BRAM memory.

\section{MBU ANALYSIS FOR CRAM AND BRAM MEMORIES}

Fig. 6 depicts a detailed distribution of the MBU events by size for CRAM. This information is important when selecting the repairing (scrubbing) method of these MBUs. As an example if the Xilinx Soft Error Mitigation (SEM) IP [13] is used, the detection and correction effectiveness is presented in Table III. When using only the ECC from frames, the SEM IP can detect up to 2 upsets per frame and correct at most 1 upset per frame. However, when selecting the mode that uses ECC and CRC codes, the SEM IP can detect and correct up to 2 upsets per frame.

In Table IV the distribution of events for BRAM is presented. Two analyses are showed. The first one analyzes the MBUs from the frame point of view. So, a multiple upset event is considered when more than one upset happens in a
BRAM frame. On the other hand, in the second analysis the MBUs are evaluated from the user data point of view. In this analysis is considered that each BRAM memory Block is used as a $36 \mathrm{~Kb}$ memory with 64 data bits word and $8 \mathrm{ECC}$ bits for each word. Since the user bits have data interleaving of 4 bits inside the BRAM frames, most of the MBU events presented in the frame point of view analysis are transformed into a 1 upset per word in the user data point of view analysis.

Comparing the results from CRAM and BRAM, the LET dependence in both types of frames has a clearly different effect.

\section{INVESTIGATION OF UPSET SENSITIVITY by SEE MODELING}

MUSCA SEP3 is a soft error prediction tool based on a Monte-Carlo approach, which performs a full flow of simulations from the interaction of the radiation particles with 


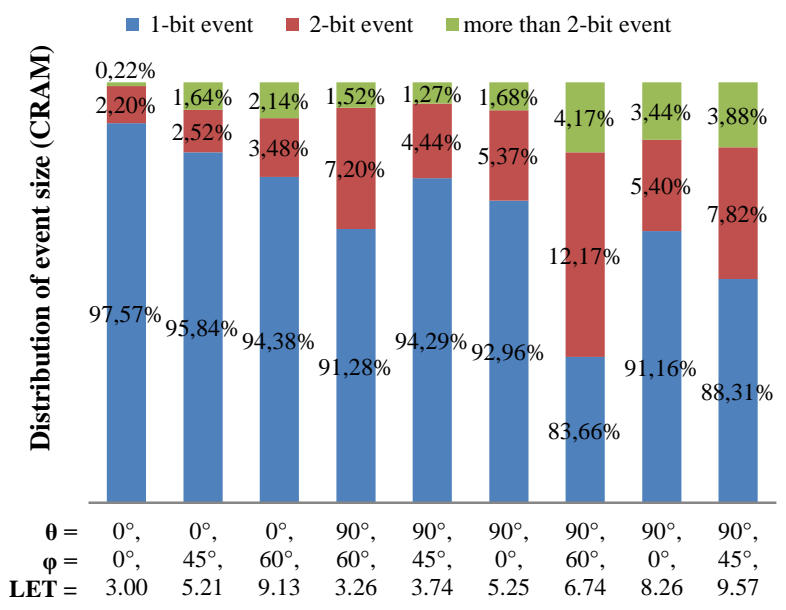

Fig. 5. Distribution of events by size for CRAM.

TABLE IV

EFFECTIVENESS FOR DETECT AND REPAIR UPSETS IN CRAM

\begin{tabular}{ccccr}
\hline \hline \multirow{2}{*}{ Run } & \multicolumn{2}{c}{ Xilinx SEM IP (ECC only) } & \multicolumn{2}{c}{ Xilinx SEM IP (ECC + CRC) } \\
\cline { 2 - 5 } & Detection & Correction & Detection & Correction \\
\hline 1 & $99.78 \%$ & $97.57 \%$ & $99.78 \%$ & $99.78 \%$ \\
2 & $98.36 \%$ & $95.84 \%$ & $98.36 \%$ & $98.36 \%$ \\
3 & $97.86 \%$ & $94.38 \%$ & $97.86 \%$ & $97.86 \%$ \\
4 & $98.48 \%$ & $91.28 \%$ & $98.48 \%$ & $98.48 \%$ \\
5 & $98.73 \%$ & $94.29 \%$ & $98.73 \%$ & $98.73 \%$ \\
6 & $98.32 \%$ & $92.96 \%$ & $98.32 \%$ & $98.32 \%$ \\
7 & $95.83 \%$ & $83.66 \%$ & $95.83 \%$ & $95.83 \%$ \\
8 & $96.56 \%$ & $91.16 \%$ & $96.56 \%$ & $96.56 \%$ \\
9 & $96.12 \%$ & $88.31 \%$ & $96.12 \%$ & $96.12 \%$ \\
\hline \hline
\end{tabular}

TABLE III

DISTRIBUTION OF EVENTS BY SIZE FOR BRAM

\begin{tabular}{crrrrrr}
\hline \hline \multirow{2}{*}{ Run } & \multicolumn{3}{c}{ Frame View } & \multicolumn{4}{c}{ User Data Word View } \\
\cline { 2 - 7 } & $\begin{array}{c}\mathbf{1} \\
\text { upset }\end{array}$ & $\mathbf{2}$ upsets & $\begin{array}{c}\mathbf{>} \\
\text { upsets }\end{array}$ & $\begin{array}{c}\text { 1 } \\
\text { upset }\end{array}$ & $\begin{array}{c}\mathbf{2} \\
\text { upsets }\end{array}$ & $\begin{array}{c}>\mathbf{2} \\
\text { upsets }\end{array}$ \\
\hline 1 & $97.97 \%$ & $1.94 \%$ & $0.09 \%$ & $98.34 \%$ & $1.63 \%$ & $0.03 \%$ \\
2 & $98.44 \%$ & $1.42 \%$ & $0.15 \%$ & $100.00 \%$ & $0.00 \%$ & $0.00 \%$ \\
3 & $81.34 \%$ & $18.54 \%$ & $0.12 \%$ & $99.87 \%$ & $0.13 \%$ & $0.00 \%$ \\
4 & $100.00 \%$ & $0.00 \%$ & $0.00 \%$ & $100.00 \%$ & $0.00 \%$ & $0.00 \%$ \\
5 & $98.95 \%$ & $1.02 \%$ & $0.03 \%$ & $99.13 \%$ & $0.87 \%$ & $0.00 \%$ \\
6 & $99.04 \%$ & $0.93 \%$ & $0.03 \%$ & $99.35 \%$ & $0.65 \%$ & $0.00 \%$ \\
7 & $99.04 \%$ & $0.92 \%$ & $0.04 \%$ & $99.28 \%$ & $0.68 \%$ & $0.04 \%$ \\
8 & $99.83 \%$ & $0.17 \%$ & $0.00 \%$ & $99.98 \%$ & $0.02 \%$ & $0.00 \%$ \\
9 & $99.72 \%$ & $0.28 \%$ & $0.00 \%$ & $99.97 \%$ & $0.03 \%$ & $0.00 \%$ \\
\hline \hline
\end{tabular}

the device down to the occurrence of the soft error in the circuit (in this work the SRAM based FPGAs). The complete principle of the modeling is reported in previous works [3][5]. These simulations use nuclear database and take into account the dynamic transport and charge collection mechanisms (multi-collections, charges sharing pulse quenching and other effects), bipolar amplification, the bias voltage, the layout, and the fabrication process with the aim to build a SET database. Next, this transient currents database is injected on each node of the cells at transistor level for an electrical transient simulation using SPICE (Simulation Program with Integrated Circuit Emphasis) simulator [16] with the aim to estimate the soft error response of the circuit. For this work, the n-MOS and p-MOS model cards are provided by PTM (Predictive Technology Model) [18].
However, because of process variations from a foundry to another, those models card need to be calibrated with the aim to fit with the technology used in the SRAM-based FPGA of the Artix-7. In addition to process variations, the main difference at layout level of those two technologies is the SRAM cell area: $0.155 \mu \mathrm{m}^{2}$ and $0.127 \mu \mathrm{m}^{2}$ for the LP and HP respectively. Xilinx uses the TSMC HPL process. The HPL process was developed for Xilinx exclusively by TSMC. The characteristics of HPL process are "close" to LP process but with higher performances. Because of confidential reasons, the HPL process is not accessible for this work. Then, in this section, the impact of the process on the upset cross section is investigated considering two 28-nm TSMC processes: the Bulk 28-nm Low Power (LP) technology and the 28-nm low power with high-k metal gates (HP) technology [19]. Hypotheses have been done for the information related to transistor sizes. These hypotheses are based on a die shrink of 45-nm technology layout published in by Luo et al [20]. The electrical performances of those technologies are different. The butterfly curves measured (in black closed squares) as a function of voltage scaling highlight this variation in terms of reliability as shown in Fig. 7.

These butterfly curves show the Static Noise Margin (SNM) in read mode and have been obtained from Shien-Yang Wu et al [17]. As mentioned, a calibration of the BSIM parameters of the n-MOS and p-MOS transistors allowed to obtain a relevant modeling of the SRAM cells. This point is required with the aim to propose a relevant soft error estimation and analysis.

Fig. 8 presents the comparison of experimental data measured at LAFN-USP (opened black squares) and issued from Gadlage et al [14] (closed black squares) and MUSCA SEP3 calculations coupled with electrical simulations at transistor level with calibrated model cards for the technologies, $0.127 \mu \mathrm{m}^{2}$ and $0.155 \mu \mathrm{m}^{2}$ i.e., LP (closed red dots) and HP (closed blue triangles) respectively.
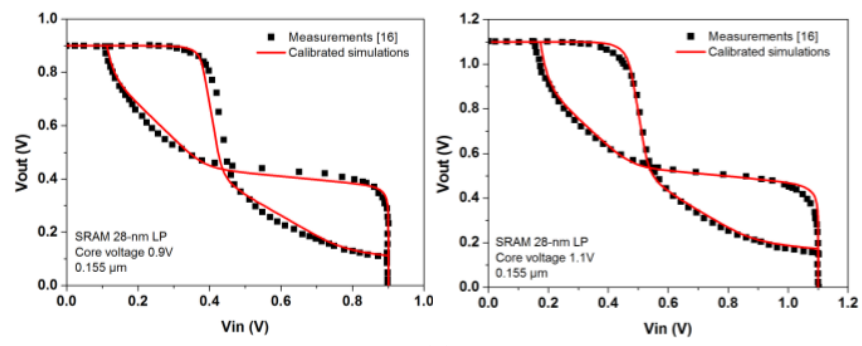

(a)
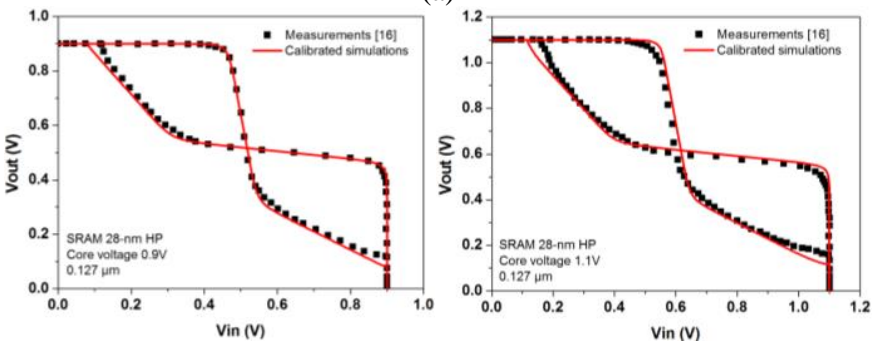

(b)

Fig. 7. Static Noise Margin in read mode, (a) 28-nm Low Power (LP) technology, (b) the 28-nm low power with high-k metal gates (HP) technology [17]. 
The comparisons are done for heavy ions data with a range of LET from $0.1 \mathrm{MeV} \cdot \mathrm{cm}^{2} \cdot \mathrm{mg}^{-1}$ up to $40 \mathrm{MeV} \cdot \mathrm{cm}^{2} \cdot \mathrm{mg}^{-1}$. A good correlation is shown as well in term of LET threshold and saturation for the higher LETs. As mentioned above, the LP technology is less sensitive to SEU because of a lower SNM. The critical charge (Qcrit) depends on the gate oxide capacitance (Cox) and the static noise margin, as defined in equation (2) [21]. As reported in [22][23], the static noise margin depends on the core voltage of the SRAM cell. SRAM voltage may vary during operation, with the SRAM placed in a low voltage state during idle period to save power, and the supply voltage raised while SRAM is active.

$$
Q_{\text {crit }}=C_{o x} \times S N M
$$

The higher sensitivity of HP process can be explained by the diffusion mechanism of the generated charges which disturbs the nominal behavior (especially in hold mode) of the struck and adjacent memory cells.

This change in the SEU sensitivity between HL and LP is limited at the threshold because of the very low LET threshold of the two processes. While at saturation, the higher multiple cell upsets ratio of HP technology induce a higher SEU cross section than the LP technology.

The correlation of the SEU sensitivity of the LP technology and the strong overestimation of the SEU sensitivity of the HP technology allows proposing a hypothesis on the process used by Xilinx for CRAM and BRAM in the FPGA. The TSMC HPL process used for CRAM and BRAM memories are not reachable. The SEE prediction tool has been used to determine the best representative technological process of $28 \mathrm{~nm}$ by the mean of a comparison of SEE cross section. This work shows that, the LP process calibrated with the use of SNM measurements can be used with the aim to investigate the SEE sensitivity of Xilinx FPGA Artix-7. For this reason, only the LP technology is presented in the next figures.

Fig. 9 presents the investigation of the impact of device orientation impact on the upset occurrence as a function of the effective LET obtained by simulation. The first observation is that the 28-nm SRAM cell is lightly more sensitive when the heavy ion strikes the device at $90^{\circ}$ as illustrated in Fig. 5 for the same effective LET. This point should indicate the orientation of the SRAM cell layout in the FPGA die. A second point interesting to note is the trend is highlighted for low LET. Actually the difference of upset cross section at $0^{\circ}$ and $90^{\circ}$ decreases by a factor of 3 between $6 \mathrm{MeV} . \mathrm{cm}^{2} . \mathrm{mg}^{-1}$ to $20 \mathrm{MeV} \cdot \mathrm{cm}^{2} \cdot \mathrm{mg}^{-1}$.

Fig. 10 and Fig. 11 present the heavy ion tilt on the single upset cross section for the device orientation of $90^{\circ}$ and $0^{\circ}$ respectively. It is interesting to note that an increase in the tilt of heavy ion increase the LET threshold. This point is observed for the two devices orientation. A second point, which can be observed in the simulation results, is the light decrease in the SEU cross section induced by the increase in the heavy ion tilt. This trend is highlighted especially at 10 MeV.cm². $\mathrm{mg}^{-1}$.

This result is in agreement with the trend of the experimental data presented in the first part of this work. However, the effect is not totally clear on each simulation results. A more detailed modeling of the back end of line and a more realistic modeling of the charge generation in the actives area must highlight this effect induced by the specificity of ion characteristics used in facility of the Laboratório Aberto de Física Nuclear of the Universidade de São Paulo.

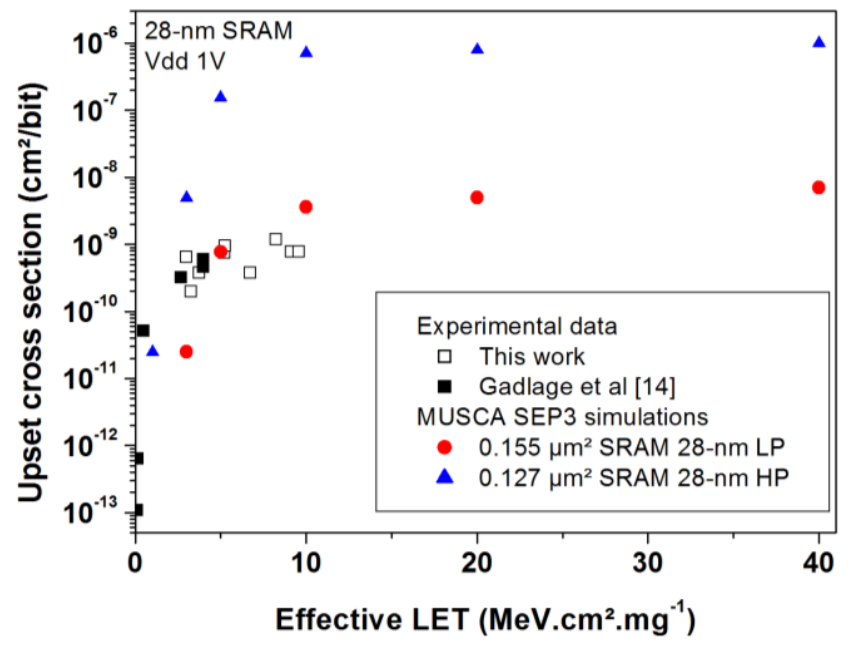

Fig. 8. Comparison of upset cross section per bit measured at LAFN-USP (opened black squares) and issued from [14] (closed black squares) and calculated by MUSCA SEP3 for the low power SRAM (closed red dots) and for the high performance SRAM (closed blue triangles) for heavy ions with a range of LETs from 0.1 up to $40 \mathrm{MeV} \cdot \mathrm{cm}^{2} \cdot \mathrm{mg}^{-1}[1]$.

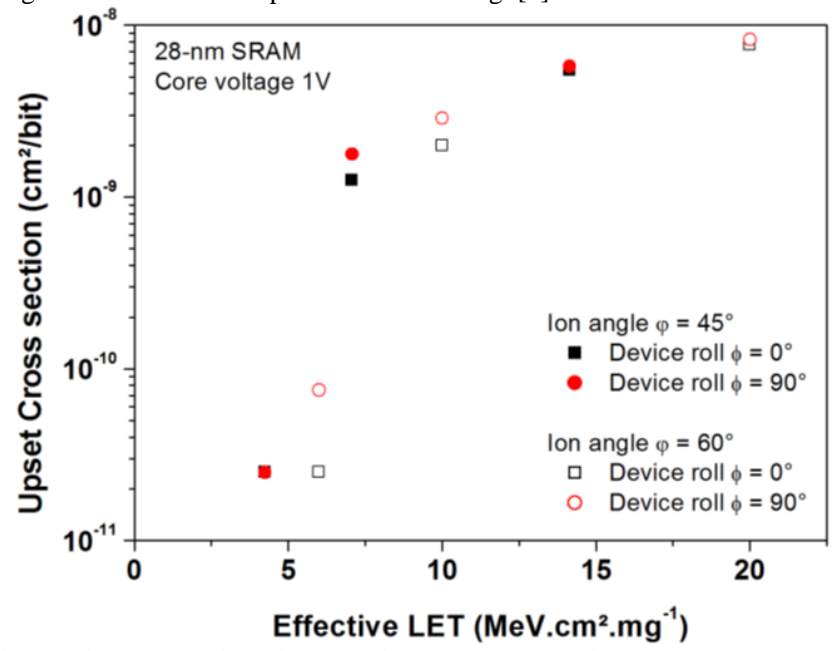

Fig. 9. Simulation of the impact of $28 \mathrm{~nm}$ SRAM orientation on upset cross section as a function of ion angles for a range of LET.

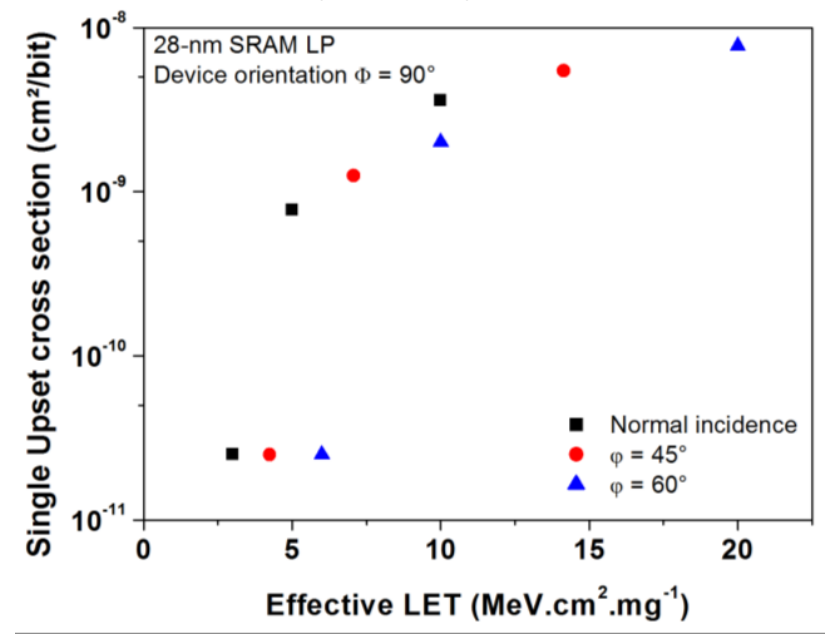

Fig. 10. Simulation of the impact of heavy ion angle on the SEU cross section considering $90^{\circ}$ of device orientation. 


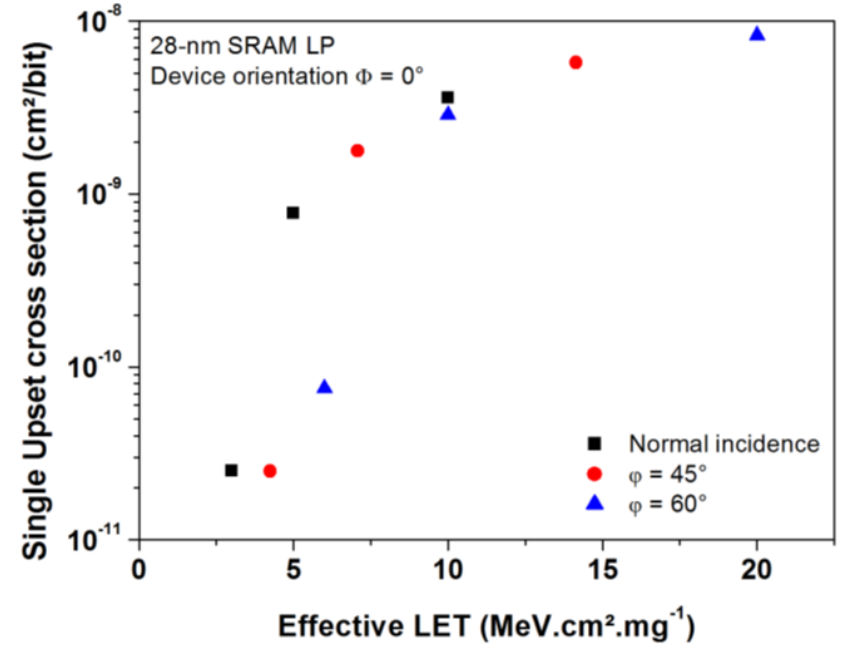

Fig. 11. Comparison of the simulation results of the impact of heavy ion angle on the SEU cross section considering $0^{\circ}$ of device orientation [1]

\section{CONCLUSIONS}

This work presents the impact of low LET heavy ions particles on the reliability of 28-nm Bulk SRAM cells from Artix-7 FPGA. New radiation tests considering the rotation of the device has been presented. The analysis of the radiation tests showed significant differences in the MBU cross section of CRAM and BRAM memory cells under various angles of rotation of the device. This analysis has been compared to previous data dedicated to the impact of the angles of incidence. It has been shown that MBU events are influenced not only by the LET of the particle but also the trajectory of the particle when it hits the device. The simulation results obtained with MUSCA SEP3 tool showed a very good agreement between the prediction and the measurements, as well the comparisons with previous publications. The TSMC HPL process used for CRAM and BRAM memories are not reachable. The SEE prediction tool has been used to determine the best representative technological process of $28 \mathrm{~nm}$ by the mean of a comparison of SEE cross section. This work shows that, the LP process calibrated with the use of SNM measurements can be used with the aim to investigate the SEE sensitivity of Xilinx FPGA Artix-7; for this reason, the LP process have been used to analyze the effect of DUT orientation presented in experimental results. MUSCA SEP tool has been used to highlight that the angle configuration as a function of the layout of the memory cell impact strongly the upset sensitivity of CRAM and BRAM used in the FPGA Artix-7.

\section{REFERENCES}

[1] J. Tonfat, F. Lima Kastensmidt, L. Artola, G. Hubert, N. H. Medina, N. Added, V. A. P. Aguiar, F. Aguirre, E. L. A. Macchione, M. A. G. Silveira, "Analyzing the Influence of the Angles of Incidence and Rotation on MBU Events Induced by Low LET Heavy Ions in a 28 -nm SRAM-based FPGA", in Proc. 16th Eur. Conf. Radiation and Its Effects on Components and Systems, 2016. to be published.

[2] H. Quinn, P. Graham, J. krone, M. Caffrey, and S. Rezgui, "Radiationinduced multi-bit upsets in SRAM-based FPGAs," IEEE Trans. Nucl. Sci., vol. 52, no. 6, pp. 2455-2461, Dec, 2005.
[3] G. Hubert, S. Duzellier, C. Inguimbert, C. Boatella-Polo, F. Bezerra and R. Ecoffet, "Operational SER Calculations on the SAC-C Orbit Using the Multi-Scales Single Event Phenomena Predictive Platform (MUSCA SEP3)," IEEE Trans. Nucl. Sci., vol. 56, no. 6, pp. 3032-3042, Dec. 2009.

[4] L. Artola, G. Hubert, K. M. Warren, M. Gaillardin, R. S. Schrimpf, R. A. Reed, R. A. Weller, J. R. Ahlbin, P. Paillet, M. Raine, S. Girard, S. Duzellier, L.W. Massgengill, and F. Bezerra, "SEU Prediction From SET Modeling Using Multi-Node Collection in Bulk Transistors and SRAMs Down to the $65 \mathrm{~nm}$ Technology Node," IEEE Trans. Nucl. Sci., vol. 58, no. 3, pp. 1338-1346, June 2011.

[5] G. Hubert and L. Artola, "Single-Event Transient Modeling in a 65-nm Bulk CMOS Technology Based on Multi-Physical Approach and Electrical Simulations," IEEE Trans. Nucl. Sci., vol. 60, no. 6, pp. 44214429, Dec. 2013.

[6] Nexys 4 Board Reference Manual, Digilent, 1300 Henley Ct. Pullman, WA 99163 , Sept. 2013.

[7] D. White, "Considerations Surrounding Single Event Effects in FPGAs, ASICs, and Processors". [Online], 2012. Available: http://www.xilinx.com/support.html\#documentation.

[8] V.A.P. Aguiar, N. Added, N.H. Medina, E.L.A. Macchione, M.H. Tabacniks, F.R. Aguirre, M.A.G. Silveira, R.B.B. Santos, L.E. Seixas Jr., "Experimental Setup for Single Event Effects at Sao Paulo Pelletron Accelerator" Nucl. Inst. Meth. Phys. Res. B., 332, p. 397-400, 2014

[9] J. F. Zeigler, M. D. Zeigler, and J. P. Biersack, Stopping and Range of Ions in Matter SRIM [Online]. 2013. Available: http://www.srim.org/.

[10] Xilinx Inc., iMPACT Help [Online]. 2009. Available: http://www.xilinx.com/support/documentation/sw_manuals/xilinx11/ise help_start.htm.

[11] M. Wirthlin, D. Lee, G. Swift and H. Quinn, "A Method and Case Study on Identifying Physically Adjacent Multiple-Cell Upsets Using 28-nm, Interleaved and SECDED-Protected Arrays," IEEE Trans. Nucl. Sci., vol. 61, no. 6, pp. 3080-3087, Dec. 2014.

[12] H. Tausch, "Simplified birthday statistics and Hamming EDAC," IEEE Trans. Nucl. Sci., vol. 56, no. 2, pp. 474-478, Apr. 2009.

[13] Xilinx Inc., LogiCORE IP Soft Error Mitigation Controller v4.0, 2013.

[14] M. J. Gadlage, A. H. Roach, A. R. Duncan, M. W. Savage and M. J. Kay, "Electron-Induced Single-Event Upsets in 45-nm and 28-nm Bulk CMOS SRAM-Based FPGAs Operating at Nominal Voltage," IEEE Trans. Nucl. Sci., vol. 62, no. 6, pp. 2717-2724, Dec. 2015.

[15] H. Quinn, K. Morgan, P. Graham, J. Krone, and M. Caffrey, "Static Proton and Heavy Ion Testing of the Xilinx Virtex-5 Device," in Proc. IEEE Radiation Effects Data Workshop, 2007, pp. 177-184.

[16] L. Nagel and D. O. Pederson, SPICE (Simulation Program with Integrated Circuit Emphasis), Memorandum No. ERL-M382, University of California, Berkeley, Apr. 1973.

[17] Shien-Yang Wu et al., "A highly manufacturable 28nm CMOS low power platform technology with fully functional $64 \mathrm{Mb}$ SRAM using dual/tripe gate oxide process," in Proc. Symposium on VLSI Technology, Honolulu, HI, 2009, pp. 210-211.

[18] Predictive Technology Model. [Online]. Available: http://ptm.asu.edu/

[19] S. Dixon-Warren "A Review of TSMC $28 \mathrm{~nm}$ Process Technology" Dec. 2012. [Online]. Available: https://www.chipworks.com/aboutchipworks/overview/blog/review-tsmc-28-nm-process-technology.

[20] Z. Luo, N. Rovedo, S. Ong, B. Phoong, M. Eller, H. Utomo, C. Ryou, H. Wang, R. Stierstorfer, L. Clevenger, S. Kim, J. Toomey, D. Sciacca, J. Li, W. Wille, L. Zhao1, L. Teo1, T. Dyer, S. Fang, J. Yan, O. Kwon, O. Kwon, D. Park, J. Holt, J. Han, V. Chan, J. Yuan, T. Kebede, H. Lee, S. Kim, S. Lee, A. Vayshenker, Z. Yang, C. Tian, H. Ng, H. Shang, M. Hierlemann, J. Ku, J. Sudijono1, M. Ieong, "High performance transistor featured in an aggressively scale 45nm Bulk CMOS technology", in Proc. IEEE Symposium on VLSI Technology, pp. 2-3, 2007.

[21] M. P. King, R. A. Reed, R. A. Weller, M. H. Mendenhall, R. D. Schrimpf, M. L. Alles, E. C. Auden, S. E. Armstrong, and M. Asai, "The impact of delta-rays on single event upsets in highly scaled SOI SRAMs", IEEE Trans. Nucl. Sci., vol 57, no. 6, pp. 3169-3175, Dec. 2010.

[22] S. M. Jahinuzzaman, "Modeling and mitigation of soft error in Nanoscale SRAMs", PhD manuscript, University of Waterloo, Ontario, Canada, 2008.

[23] A. Pavlov, et al, "An SRAM weak cell fault model and a DFT technique with a programmable detection threshold," in Proc. IEEE Int. Test Conf. (ITC), pp. 1006 - 1015, 2004. 